\title{
A Computational Method Involving Surface Area to Volume Ratio to Estimate Inorganic Nanoparticle Efficacy
}

\author{
Wesley A. Williams ${ }^{1,2,}$, Ashley J. Denslow ${ }^{1,2}$, Peter W. Radulovic ${ }^{1,2}$, Daniel J. Denmark ${ }^{1,2}$, and Shyam S. Moha- \\ patra ${ }^{1,2,3,4}$ \\ 1 Graduate Programs, Taneja College of Pharmacy, University of South Florida, Tampa, FL; \\ 2 USF Division of Translational Medicine and Center for Research and Education in Nanobioengineering, \\ University of South Florida, Tampa, FL; \\ 3 Department of Internal Medicine, Morsani College of Medicine, University of South Florida, Tampa, FL; \\ 4 James A Haley VA Hospital, Tampa, FL; \\ * Correspondence: smohapat@usf.edu and wesleyallenwilliams@usf.edu
}

\begin{abstract}
Inorganic nanoparticles are utilized for therapeutic, diagnostic, or in combination, theranostic purposes. The latter involves simultaneous sensing, imaging, or tracking of drug delivery. Furthermore, these nanoparticles can differ in their morphologies, which affect outcomes such as the effectiveness of hyperthermia, induction, drug loading, circulation time by escaping the body's immune system, imaging modality clarity, and biosensing. However, design of these theranostics is limited by the lack of a method to predict their therapeutic efficacy. Herein, we report a simple and novel computational approach via algebraic and geometric calculations of surface area $(\mathrm{SA})$ to volume $(\mathrm{V})$ ratios $(\mathrm{SA}: \mathrm{V})$ which can help predict the efficacy of the inorganic nanoparticles of the investigated morphologies. The approach comprises a coding platform for the program and uses Python 3 on a Windows 10 operating system. Analyses of 29 polyhedral morphologies that inorganic nanoparticles could assume ex silico showed that only particular concave and convex morphologies in this size regime are more productive over the standard sizes as well as a few noted in literature for baseline comparison. Our results provide a method that can aid in predicting the efficacy of inorganic nanoparticles with certain morphology giving rise to their fundamental basis and eventual implementation ex silico.
\end{abstract}

Keywords: inorganic nanoparticles; in silico; optimization; theranostic; therapeutic; diagnostic; computation; coding; Python

\section{Introduction}

Inorganic nanoparticles have been used across three main nanotherapeutic modes as a methodology for optimizing medicine and testing in terms of cost, ease of use, accessibility, and toxicity: therapeutic, diagnostic, and theranostic. This particular branch of medicine's interdisciplinary nature and versatility has made it an attractive target in the aforementioned modes of delivery. Inorganic particles, like any other innovation, must be tunable and amenable to further optimization to progress past the state-of-the-art via physicochemical processes that achieve this effect. Hypothetically speaking, they may offer an increase in efficacy in terms of tuning the physical property of SA:V alone. Henceforth, this paper aims to explore the current state-of-the-art of inorganic nanoparticles in regard to their function, how researchers synthesize these nanoformulations, their direct efficacy in basic, preclinical, and clinical research and finally, how the 29 morphologies tested in this paper could further the efficacy of current research in nanomedicine.

Concerning the state-of-the-art, in a therapeutic sense, for example, nanoparticles' surface chemistry can be altered for conjugating drug payloads and biological molecules for drug delivery, offering antimicrobial measures of protection, and even as vaccine carriers (Pfizer/BioNTech's and Moderna's SARS-CoV-2 nano vaccines) [1,2]. In the diagnostic sense, for example, these nanoparticles can serve as label-free contrast agents that 
produce a higher resolution image of cancers that may escape traditional metal-based dyes. They can also serve as sensors that monitor levels of key diagnostic biomarkers in human sera (like molecularly imprinted nanoparticles for point-of-care-testing (POCT)), potentially facilitating high-level care more ubiquitously in comparison to traditional testing via their selectivity for specific target molecules. Their reusability, sensitivity through increasing conduction physiochemically, portability, and reproducibility all aid in that endeavor and purpose [3]. Lastly, in the theranostic sense, these nanoparticles, in addition to identifying cancerous cells via imaging, can exact localized hyperthermia without inducing damaging thermal ablation to surrounding tissues via alternating magnetic frequencies. Some metal/metal oxide nanoparticles exhibit a paramagnetic or superparamagnetic nature enabling their susceptibility to AMFs and resultant heat generation. One such example uses superparamagnetic iron-oxide nanoparticles (SPIONs) encased in a photopolymerizable substance poly-N-isopropylacrylamide (PNIPAM) that can release these nanoparticles on exposure to an alternating magnetic field, ensuring a more optimized usage and less ability to agglomerate and trigger an immune response [4-6].

Inorganic nanoparticles can be fabricated in a variety of morphologies aside from the traditional sphere or spheroid. Tuning inorganic nanoparticles in a physical sense allows researchers to increase their efficiency with ease, allowing them to improve their absolute loading capability and efficiency, magnetizability, and increased reactivity making them more prone to interact with biological contaminants as an antibiotic electrochemically, for example. Multitudes of examples occur in literature that showcase the efficacy of inorganic nanoparticles with exotic morphology from basic to preclinical to clinical trial research further underscoring the importance of investigating fundamental parameters and properties.

In the basic research sense, for example, Kordashenas and Ghorbani reported the reactivities of different morphological applications of silver nanoparticles like the cube, sheet, and near-spheroid nanoparticle elucidating that a significant increase in efficacy is shown giving a potential rise as a nanoformulation made for antimicrobial purposes [7]. Furthermore, it was also shown that increasing the particle's surface area (SA) to volume $(\mathrm{V})$ ratio $(\mathrm{SA}: \mathrm{V})$ at a standardized size was suggested increased efficacy of nanoparticles nanotoxicological effects [8]. Moreover, octahedral iron oxide nanoparticles, in particular, were shown to enhance magnetically induced hyperthermia even more so than traditionally spheroid nanoparticles [9].

Aside from efficacy at the basic research level it is important to underscore the techniques associated with fabricating nanoformulation and potential nanoparticles with exotic morphologies at the same level. The state-of-the-art in bottom-up and top-down processes will be showcased before we move onto higher level research examples of inorganic nanoparticles with exotic morphologies for foundational purpose.

Nanoparticles with diverse morphologies can be produced by different chemical means from the bottom up via crystallization or seeded-nucleation techniques [10]. Thus, Thambi and associates reported creating multiple morphologies for gold nanorods by changing the concentration of silver nitrate in the presence of varying $\mathrm{pH}$ levels, most notably for creating octahedral nanoparticles [11]. Also, an approach for octahedral nanoparticles and other morphologies such as truncated cubes, cuboctahedra, and truncated octahedra were reported to develop as the plane encompassing the $\mathrm{x}, \mathrm{y}$, and $\mathrm{z}$ direction is formed at different convective solvent flows and vertex truncation [12]. Furthermore, a bottom-up process of core-shell type Pt-Pd icosahedral nanoparticle formulation that implements multiphase (air/liquid) microfluidics employing sodium tetrachloropalladate $\left(\mathrm{Na}_{2} \mathrm{PdCl}_{4}\right)$ and PVP dispersed diethylene glycol solution was reported [13]. Moreover, a method of creating concave polyhedral specifically tris-octahedral nanoparticles, was shown by adding a higher concentration of reductants (like halide anions), which tempered the formation of gold nanoparticles with desired morphology [14]. Lastly, the methodologies for creating multiple types of morphologies including icosidodecahedral, dodecahedral, and tetrahedral inorganic particles using seeded-growth (former two)/oleylamine-mediated protocols has been reviewed and detailed extensively[15]. 
In addition to the above referred bottom-up approaches, several top-down approaches have been investigated. A recent review details several popular methods. One such as optical lithography, a nanofabrication tool with longevity, can quickly form 3D structure at decent resolutions in a high throughput manner. The only issue is that there is a tradeoff that must be decided between resist sensitivity and resolution. E-beam lithography allows etching of small features less than $20 \mathrm{~nm}$ in dimension. Unfortunately, it is a highly expensive and low throughput process that cannot create features less than 5 nanometers. Another method involves soft/nanoimprint lithography, which is a simple pattern transfer based nanofabrication tool capable of etching features less than $10 \mathrm{~nm}$ but has a disadvantage with its low-throughput process being unable to make densely-packed nanostructures (low periodicity). Moreover, it is costly and dependent on other means for template creation. Block co-polymer lithography is a high-throughput, low-cost method for mass producing nanostructures like spheres and cylinders but possesses issues with periodicity. Lastly, and arguably the most efficacious, scanning probe lithography can be employed: a high-resolution chemical, molecular, and mechanical nanopatterning method that has the capability of manipulating individual atoms. The caveat lies in not being a high throughput process, high expense, and requirement of an ultra-high-vacuum based version [16].

From the foundation, we can see that inorganic nanoparticles with less commonly known morphologies have been shown to possess great efficacy at higher stages of research. In the preclinical sense, a few studies have been isolated to show the relevance of morphological shape in the efficacy of the three main modes of function inorganic nanoparticles possess. One such study found that the use of prolate (1D stretch) ellipsoidal nanoparticles exhibited greater macrophage evasion in vivo and greater biodistribution in vivo suggesting a longer therapeutic window and escape potential from the reticularendothelial system (RES) compared to spherical analogs [17]. Moreover, on a morphology built to escape the reticular-endothelial system (RES) and to possess higher capability of surface modification, gold nanorods conjugated with HER2 antibodies on their surface were shown to associate with HER2 positive cell lines and tumors in murine models with high efficacy compared to the more traditional spheroid nanoparticles when considering their negative effects in general histological studies of inflammation in the liver [18,19]. For cellular uptake, Ridolfo's group found that dexamethasone loaded worm-like biodegradable nanoparticles possessed a significant capability of internalization in retinal cells despite their constituent materials across the board [20]. As for nanoplates, one group found that there was a " $68.5 \%$ " photothermal conversion efficiency increase for triangular gold nanoplates that act as an excellent catalyst for peroxidase-like antibacterial applications in MRSA-infected wounds in vivo increasing the rationale and precedence for specialized morphological nanoparticle use in the clinic [21]. Lastly, another group's review article investigating the reactivity of decahedral gold nanoparticles notated that Yeh's group's nanoformulation had the impressive ability to detect lead ion concentrations down to $1 \mathrm{ppb}$ or $4.8 \mathrm{nM}$ vastly increasing the traditional range of lead ion sensing in SPR $\mathrm{Au}$ aggregation assays. Surface interaction and defects coordinating with lead ions and citrate ions carboxylic acid (higher binding affinity) were surmised as the rationale behind their increasing sensing ability [22,23]. Exotic morphologies often increase the reactivity of many inorganic particles and in some senses, catalytically.

In context to clinical trials only one nanoformulation with exotic morphology is in process through the FDA for treatment against advanced soft tissue sarcoma whilst obtaining a "CE mark" for commercialization in the EU: NBTXR3 or "Hensify" (Nanobiotix). In addition to its ongoing FDA clinical trial concerning sarcomas, there are 7 others currently in progress for the application against lung and prostate cancer. Nanobiotix, formulation wise, is a hafnium oxide negatively-charged phosphate coated nanoparticle that facilitates tumor cell death via electron generation via external radiation stimulated with external ionizing radiation induction [24]. The nanoformulation itself is proprietary but it can be surmised as an encapsulated icosahedral inorganic nanoparticle from Nanobiotix's depictions [25]. This function allows radiotherapy to be localized and amplified in tumor 
masses at specific locations. Hensify's efficacy was examined in a phase I FDA clinical trial where 3 out of 7 evaluable patients received a median of $40 \%$ decrease in tumor volume after direct injection. Local recurrence was not seen but distant recurrence was seen (metastasis). In terms of safety, CT scans indicated that the nanoformulation retained well inside of the tumor with no leakage into the surrounding tissues across the entire therapy. Hafnium in the urine and lungs was not detected [26].

Despite extensive research in this field, determining which of the diverse morphologies nanoparticles can assume potentially yield more efficient nanoparticles remains a major limitation of the field. To meet this unmet need, herein, we undertook an investigation of 29 polyhedral morphologies that inorganic nanoparticles could assume ex silico. We show that the estimation of physical properties of these nanoparticles, such as SA:V by a computational approach, provides a key index for which the functional efficacy of inorganic nanoparticles can be measured. We hope this study, outlining the creation, potential efficacy, and modeling process presents itself as a fundamental basis for another starting point of optimizing nanoformulations.

\section{Materials and Methods}

The Processor: The processor native to the 64-bit operating system is the Intel(R) Core(TM) i3-1005G1. The size of the RAM at 12.0 GB was sufficient for multiple processes. The operating system is Windows 10 Home.

Python: The version of Python used for the program was version: 3.8.5. The anaconda application was used to consolidate the necessary packages with the Jupyter Notebook application as the coding interface. The "Math" package was used for simple mathematical functions used for SA:V calculations in the program and "NumPy" for data manipulation into arrays for plotting. "Matplotlib pyplot" was used for plotting the resulting data of the 22 shapes across the nano-regime. Finally, "Tabulate" was used for creating the table of values calculated for each shape's SA:V at $10 \mathrm{~nm}$.

Figure 1 describes the logic of our algorithm. The beginning of the code requires three user inputs that determine the outcome of the initial outputs via conditional loops. The polyhedron's name, type (concave or convex), and the circumsphere diameter a researcher wants to investigate are required. Clerical mistakes and the like trip the error and terminate the loop and the program altogether. If not, the surface area, volume, and surface area to volume ratio are calculated via mathematical derivations that equate concave polyhedra parameters as if they were convex polyhedra (details for derivations described in the Supplemental Information section) [27].

Next, the conditional loops are exited when the input conditions are given, and the calculations are outputted. Tables 2,3, and 4 and Figures 2 and 3 are outputted comparing the SA:V ratio of the various polyhedra investigated from a singular chosen circumsphere diameter and against the entire nano-regime in Figure 3. Figures 2 and 3 give visual comparisons within subgroups within convex, concave, and literature grouping against the standard circumsphere (exception for "deltoidal icositetrahedron" (mid sphere radius equation is closest to circumsphere radius versus the insphere radius equation). 


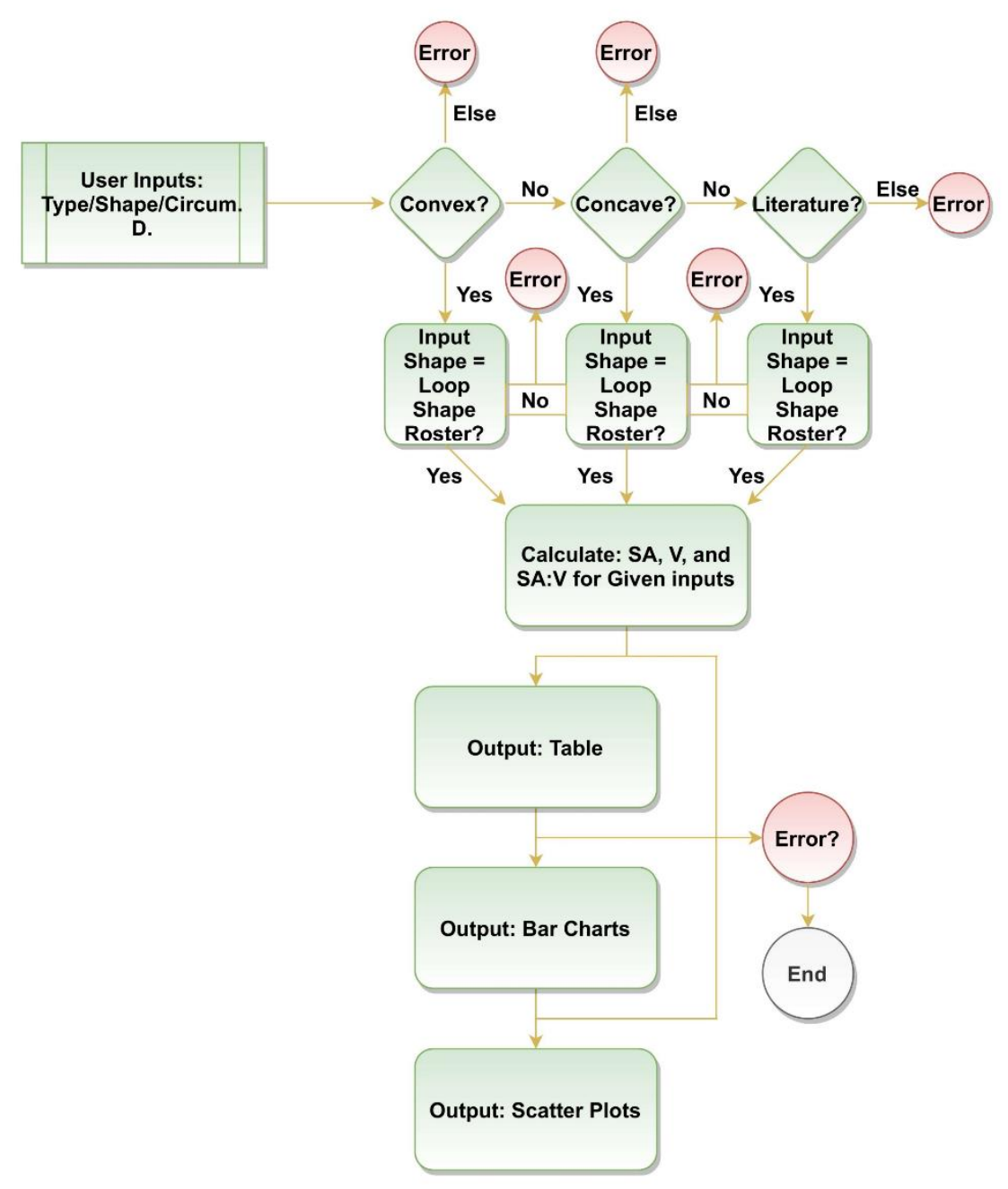

Figure 1. Logic of Mathematics Code

Statistical Analysis. Unpaired two-sample t-test was implemented. Table 5 was formatted from the analysis performed using Excel and the add-in: "Analysis ToolPak".

\section{Results}

The morphology of several convex and concave polyhedra, as well as those based in literature, were selected as a basis for investigating $\mathrm{SA}: \mathrm{V}$ with respect to a traditional sphere or spheroidal nanoparticle. These included convex polyhedra such as the Platonic, Archimedes, Catalan; concave polyhedra such as Kepler-Poinsot, Da Vinci and Wenninger, and Literature-based solids for comparison. Table 1 shows the 29 polyhedra investigated [28-40].

Many of these shapes possess unique morphologies that may potentially optimize nanoparticles' performance. Solids nearer the morphology of a sphere or spheroid nanoparticle would leave the observer to infer that their ratios are more similar in comparison to those with more dramatic constructs.

The first output from the program (Tables 2, 3, and 4) are a tabulation from all of the results of the calculations required for SA:V for each nanoparticle morphology. From inference alone it is to be expected that shapes, such as the deltoidal icositetrahedron possess an SA:V closer to a sphere than the stellated octahedron, for the inverse example, whose concavity offers more surface area against its volume at the standard diameter. For convex shapes, the number of faces on a shape seem to diminish any gain in SA:V over a sphere expectedly as, from a mathematical standpoint, it is known that as one takes the limit 
Table 1. Morphology of convex, concave, and literature-based polyhedra investigated: Convex (First Column): Tetrahedral-Dodecahedron: Platonic, Cuboctahedron-Truncated Octahedron: Archimedes, and Deltoidal Icositetrahedron: Catalan. Concave (Second Column): Great Dodecahedron-Small-Stellated Dodecahedron: Kepler-Poinsot, Stellated Octahedron: Da Vinci, and Medial Rhombic Triacontahedron-Excavated Dodecahedron: Wenninger (Abstract-Regular) Literature (Third Column) [28-40].
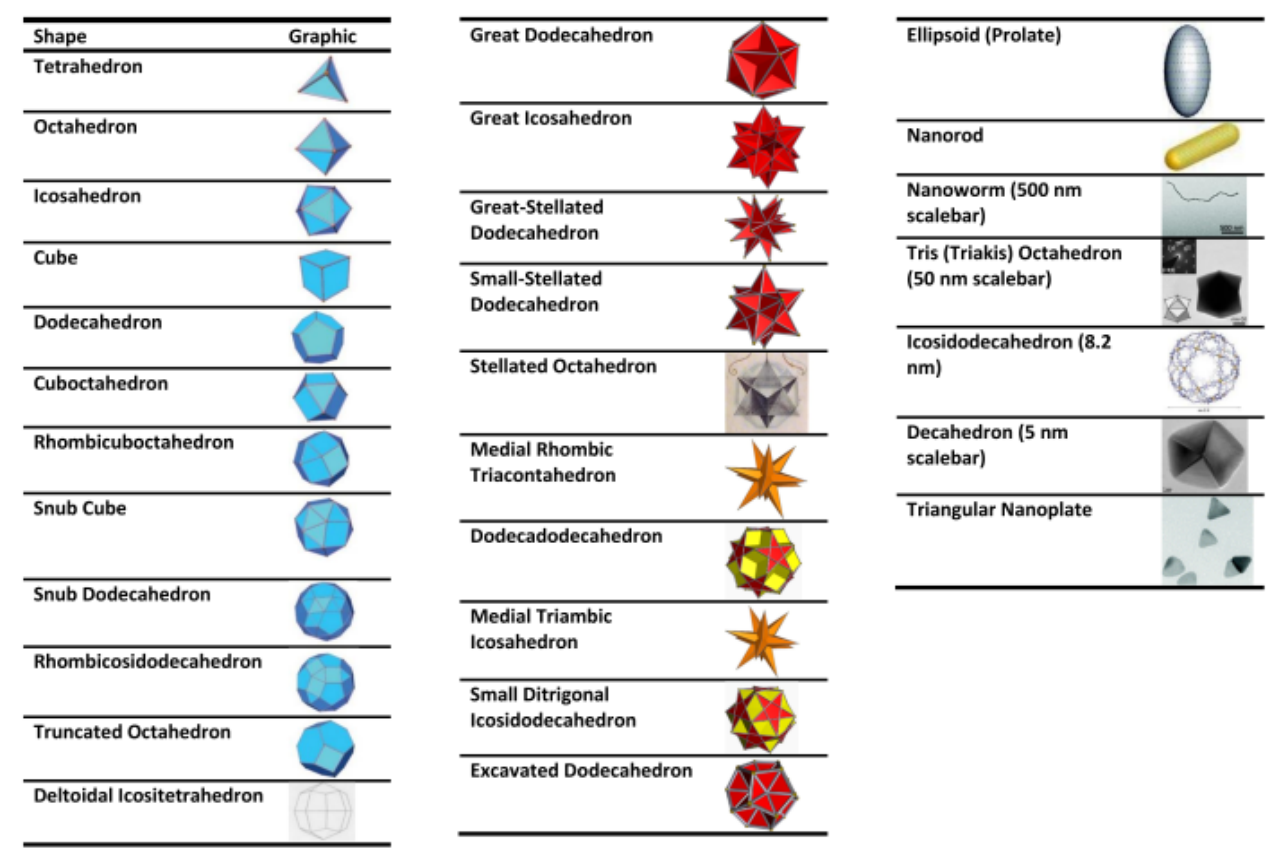

where the number of sides approaches infinity it brings a regular polygon closer to the shape of circle in the 2D sense, whereas here, the application of that limit shifts up into the third dimension with the number of faces. We see this concept well in the tetrahedron versus the rhombicuboctahedron, for example, where the increased number of faces sacrifices surface area and SA:V in the latter's case along with the icosahedron and the Archimedes solids.

Similarly, the number of faces can reduce this concept in the concave group in terms of preserved volume as evidenced by comparing the Wenninger solids with Da Vinci. Moreover, the length of a vertex to the intersection of the face in concavity contributes beneficially to a higher SA:V so long as the previous detriment is kept into consideration. We see this evidenced within Wenninger's group for the dodecadodecahedron and small ditrigonal icosidodecahedron and comparison to the medial triambic icositetrahedron. Note that the excavated dodecahedron is a remarkable case in which parts of a regular dodecahedron are cut in a regular fashion offering a substantial amount of surface area but sacrificing major volume.

Note that for the Deltoidal Icositetrahedron in the convex group the mid-sphere was calculated in absence of a calculation for the circumsphere, offering a slight discrepancy in standardization due to easier derivation. It must also be taken into account that, for a specific application, the volumes of these nanoparticles at a standard size should be recognized despite high SA:V. Again, the excavated dodecahedron looks attractive as well as the stellated octahedron but offers significantly less volume. While offering greater ability of surface modification and induction, this may be detrimental if the material itself needs to be at an adequate mass for the therapy or diagnostic capability. Surface area and volume 
Table 2. Tabulation of SA, V, and SA:V for all convex shapes with respect to input circumsphere diameter of $10 \mathrm{~nm}$ (reference sphere).

\begin{tabular}{|c|c|c|c|c|}
\hline Convex Shape (Abbrev.) & Type & $\begin{array}{c}\text { SA } \\
\left(\times 10^{-16} \mathrm{~m}^{2}\right) \\
\end{array}$ & $\begin{array}{c}V \\
\left(X 10^{-25} \mathrm{~m}^{3}\right)\end{array}$ & $\begin{array}{r}\text { SA:V } \\
\left(\times 10^{9}\right)\end{array}$ \\
\hline Tetrahedron (TE) & Platonic & 1.15 & 0.64 & 1.80 \\
\hline Octahedron (OCT) & Platonic & 1.73 & 1.67 & 1.04 \\
\hline Icosahedron (IC) & Platonic & 2.39 & 3.17 & 0.76 \\
\hline Cube (CU) & Platonic & 2.00 & 1.92 & 1.04 \\
\hline Dodecahedron (DO) & Platonic & 2.63 & 4.78 & 0.55 \\
\hline Cuboctahedron (CO) & Archimedes & 2.37 & 2.95 & 0.80 \\
\hline Rhombicuboctahedron (RCO) & Archimedes & 2.74 & 3.98 & 0.69 \\
\hline Snub Cube (SCU) & Archimedes & 2.75 & 4.06 & 0.68 \\
\hline Snub Dodecahedron (SDO) & Archimedes & 6.65 & 15.30 & 0.44 \\
\hline Rhombicosidodecahedron (RIDO) & Archimedes & 2.97 & 4.67 & 0.64 \\
\hline Truncated Octahedron (TOCT) & Archimedes & 2.68 & 3.58 & 0.75 \\
\hline Deltoidal Icositetrahedron (DIT)* & Catalan & 3.15 & 4.90 & 0.64 \\
\hline Sphere (SP) REF. & $\mathrm{N} / \mathrm{A}$ & 3.14 & 5.24 & 0.60 \\
\hline
\end{tabular}

* midsphere radius equation is closest to circumsphere radius versus the insphere radius equation should be weighed with importance and consideration in addition.

As for the literature group, all but one of the smooth solids exhibit low SA:V: the nanoworm, compared to the ellipsoid and nanorod. In terms of surface modification and external payload, the nanoworm would be highly comparable to most morphologies even more than most in the convex and concave group. With added length, by increasing the amount of pitch for the helical model of a nanoworm, we most likely would see even more $S A: V$ with higher volume for internal payloads. The ellipsoid and nanorods exhibit lower $\mathrm{SA}: \mathrm{V}$ than the circumsphere however, in literature, they show efficacy based on their unique shape in regard to immune escape and internalization showcasing that $\mathrm{SA}: \mathrm{V}$ as a sole parameter for estimating efficacy is only a start but not the entire contribution or effect for efficacy. Furthermore, the triakis octahedron and icosidodecahedron provide a slight increase in SA:V comparable to many solids in the convex group. Lastly, the triangular nanoplate and decahedron offer high SA:V efficacy over the circumsphere. 3 out of 7 shapes in this group exhibit comparably higher efficacy to those in the concave and convex group serving as a literary baseline.

Consistent with the previous tabulations, Figures 2 and 3 reveal insightful differences in SA:V against shapes within subgroups when compared to the reference sphere. The tetrahedron offers a significantly considerable amount of SA:V for nanoformulation of a standard size. The cuboctahedron, rhombicuboctahedron, and truncated octahedron offer a significantly higher $\mathrm{SA}: \mathrm{V}$ within their group though globally the significance is lost. However, what is not observed is a palpable difference in inefficacies between shapes. For 
Table 3. Tabulation of SA, V, and SA:V for all concave shapes with respect to input circumsphere diameter of $10 \mathrm{~nm}$ (reference sphere).

\begin{tabular}{|c|c|c|c|c|}
\hline Concave Shape (Abbrev.) & Type & $\begin{array}{c}\text { SA } \\
\left(\times 10^{-16} \mathrm{~m}^{2}\right)\end{array}$ & $\begin{array}{c}V \\
\left(X 10^{-25} \mathrm{~m}^{3}\right)\end{array}$ & $\begin{array}{l}\text { SA:V } \\
\left(\times 10^{9}\right)\end{array}$ \\
\hline Great Dodecahedron (GDO) & Kepler-Poinsot & 3.01 & 2.25 & 1.34 \\
\hline Great Icosahedron (GIC) & Kepler-Poinsot & 2.92 & 0.91 & 3.20 \\
\hline Great-Stellated Dodecahedron (GSDO) & Kepler-Poinsot & 5.05 & 2.37 & 2.13 \\
\hline Small-Stellated Dodecahedron (SSDO) & Kepler-Poinsot & 1.86 & 1.39 & 1.34 \\
\hline Stellated Octahedron (SOCT) & Da Vinci & 0.11 & 0.02 & 7.20 \\
\hline $\begin{array}{l}\text { Medial Rhombic Triacontahedron } \\
\text { (MRT) }\end{array}$ & $\begin{array}{l}\text { Abstract-Regular } \\
\text { (Wenninger) }\end{array}$ & 4.14 & 3.67 & 1.20 \\
\hline Dodecadodecahedron (DODO) & $\begin{array}{l}\text { Abstract-Regular } \\
\text { (Wenninger) }\end{array}$ & 1.10 & 2.57 & 0.43 \\
\hline Medial Triambic Icosahedron (MTIC) & $\begin{array}{l}\text { Abstract-Regular } \\
\text { (Wenninger) }\end{array}$ & 1.47 & 0.33 & 4.44 \\
\hline $\begin{array}{l}\text { Small Ditrigonal Icosidodecahedron } \\
\text { (SDIDO) }\end{array}$ & $\begin{array}{l}\text { Abstract-Regular } \\
\text { (Wenninger) }\end{array}$ & 1.44 & 3.36 & 0.43 \\
\hline Excavated Dodecahedron (EDO) & $\begin{array}{l}\text { Abstract-Regular } \\
\text { (Wenninger) }\end{array}$ & 3.31 & 0.19 & 17.00 \\
\hline Sphere (SP) & N/A & 3.14 & 5.24 & 0.60 \\
\hline
\end{tabular}

Kepler-Poinsot, the great icosahedron outperformed the rest though the whole offers significance against the reference sphere. For the Wenninger, we see the highest significance for the excavated dodecahedron but the aforementioned considerations still hold. For the literature group, the nanoworm and decahedron breach their group's critical threshold. The triangular nanoplate does not but is certainly comparable in the convex groups.

Significant SA:V differences are observed for nanoparticles smaller than 20 to $30 \mathrm{~nm}$, with the highest efficacy exhibited for nanoparticles less than $10 \mathrm{~nm}$ circumsphere diameter. The differential significance rate is logarithmically proportional and increases towards the picometer regime, similar to the last proportions of SA:V within subgroups. Note that the ratios of the octahedron and cube against the circumsphere are equal thus the missing curve for the octahedron is from the program's output by happenstance. Shapes with the most dramatic divergence indicate an even greater potential of efficacy towards the picometer regime.

From a statistical standpoint, the previous graphs indicate significance with respect to their respective groups determined by an unpaired two-sample t-test to indicate statically significant differences in SA:V against the reference sphere, as seen in Table 5. It was found that only the Kepler-Poinsot polyhedra group was significantly different than the other three groups (fashioned by combing the single-shaped groups from their respective shape types). However, when we look at the global t-test statistic, we see a higher significance in SA:V of the shapes against the reference sphere across the board indicating more viable shapes for experimentation. This is explained by the increased power of the test as 
Table 4. Tabulation of SA, V, and SA:V for all literature shapes with respect to input circumsphere diameter of $10 \mathrm{~nm}$ (reference sphere).

\begin{tabular}{|c|c|c|c|}
\hline Literature Shape (Abbrev.) & $\begin{array}{c}\mathrm{SA} \\
\left(\mathrm{X} 10^{-16} \mathrm{~m}^{2}\right)\end{array}$ & $\begin{array}{c}V \\
\left(X 10^{-25} \mathrm{~m}^{3}\right)\end{array}$ & $\begin{array}{c}\text { SA:V } \\
\left(\times 10^{9}\right)\end{array}$ \\
\hline Ellipsoid & 0.39 & 1.31 & 0.30 \\
\hline Nanorod & 1.18 & 4.50 & 0.26 \\
\hline Nanoworm & 0.66 & 0.10 & 6.44 \\
\hline Triakis (tris) Octahedron & 1.83 & 2.07 & 0.88 \\
\hline Icosidodecahedron & 2.80 & 4.08 & 0.69 \\
\hline Decahedron & 2.80 & 0.58 & 4.85 \\
\hline Triangular Plate & 1.62 & 0.81 & 2.00 \\
\hline Sphere (SP) & 3.14 & 5.24 & 0.60 \\
\hline
\end{tabular}

driven by the increased sample size.

In addition to those elucidated by the bar graph figures, all but the Dodecadodecahedron and small ditrigonal icosidodecahedron from the concave group are statistically significant enough for consideration versus none of the shapes in the convex category, meeting the critical SA:V to declare it. Moreover, it is important to note that shapes shown in the "within group" t-tests elucidate this better than the global for the group as it pulls less weight in terms of the global significance. This fact applies inversely in the concave category since the group pulls the highest weight of significance, thereby giving importance to the "global" $t$-test for further elucidation. The literature group's variance and mean are similar to the global $\mathrm{t}$-test offering the least effect.

The first assumption with the choice of the test type is that the sample size is adequate. The second assumption is that the data is assumed to possess normality due to experimenter's aiming for a mean size in a colloidal or nanofabricated batch of nanoparticles making the mean observation population based. The means calculated are from continuous or ordinal within the tests. The third assumption is the homogeneity of variance which is more difficult to ascertain based on multiple variables like the fabrication methods, material type, and researchers themselves. The second falls under the issues of the third assumption as well when it comes to possible ascertainment issues. The fourth assumption is that the alpha at 0.05 is established as a decent parameter for the critical p-value. The fifth and final assumption is the choice of randomness. Arguably, the choice of $10 \mathrm{~nm}$ may be non-random as it is within the nano-regime and the assumed population mean of a 

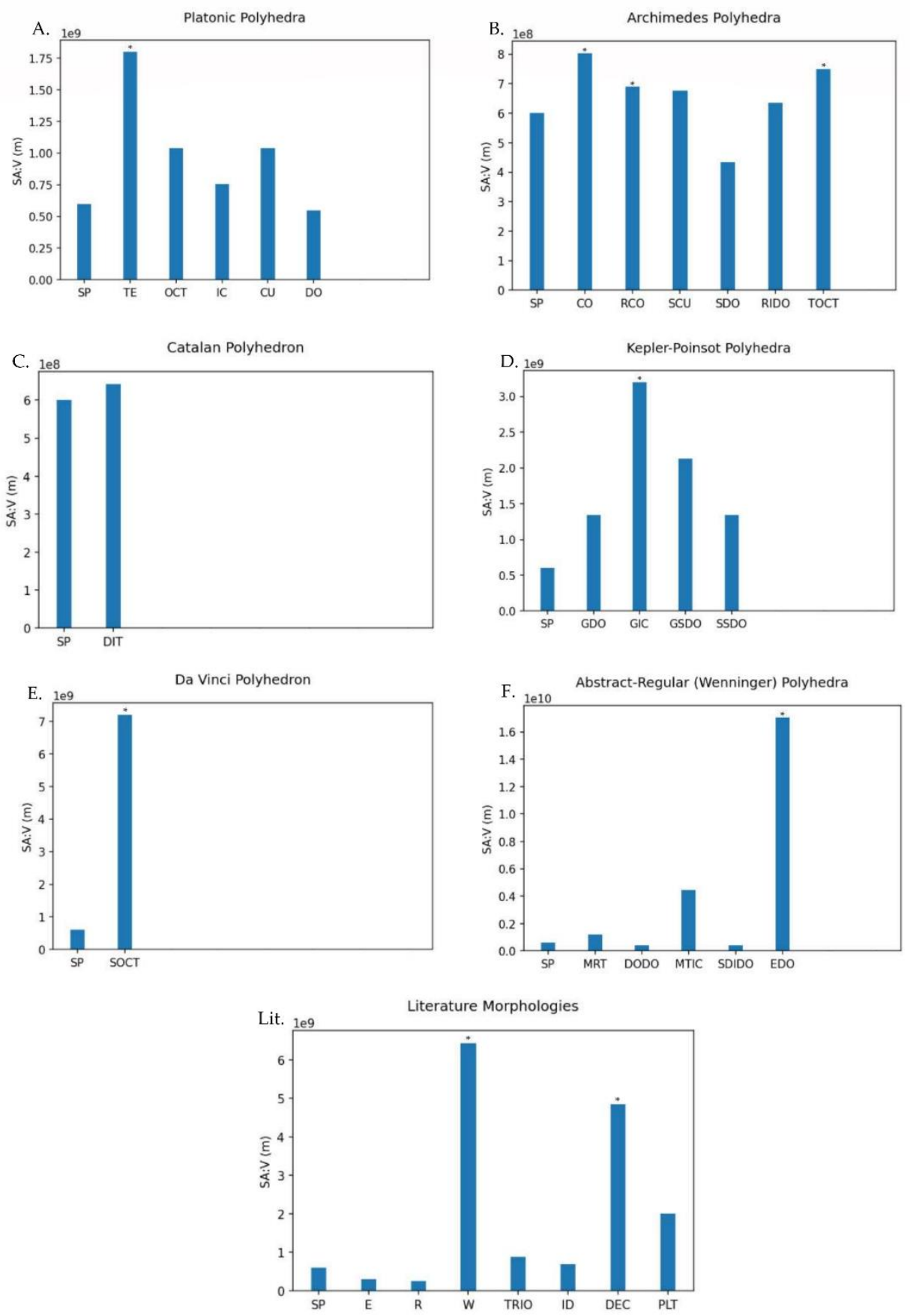

Figure 2. SA:V, measured in $\mathrm{m}^{-1}$, as a function of morphology for the A. Platonic, B. Archimedes, C. Catalan, D. Kepler-Poinsot, E. Da Vinci, F. Wenninger polyhedra, and Lit. Literature Solids. Note the multiplicative scaling factor is given at the top of the $\mathrm{SA}: \mathrm{V}$ axis (Asterisks denote "experimenter's mean or chosen value" that surpasses a group's critical value on unpaired 2-sample t-tests).

colloi-

dal or batch nanoparticle solution but the variety of shapes and their unique calculations may give more credence to the assumption. 

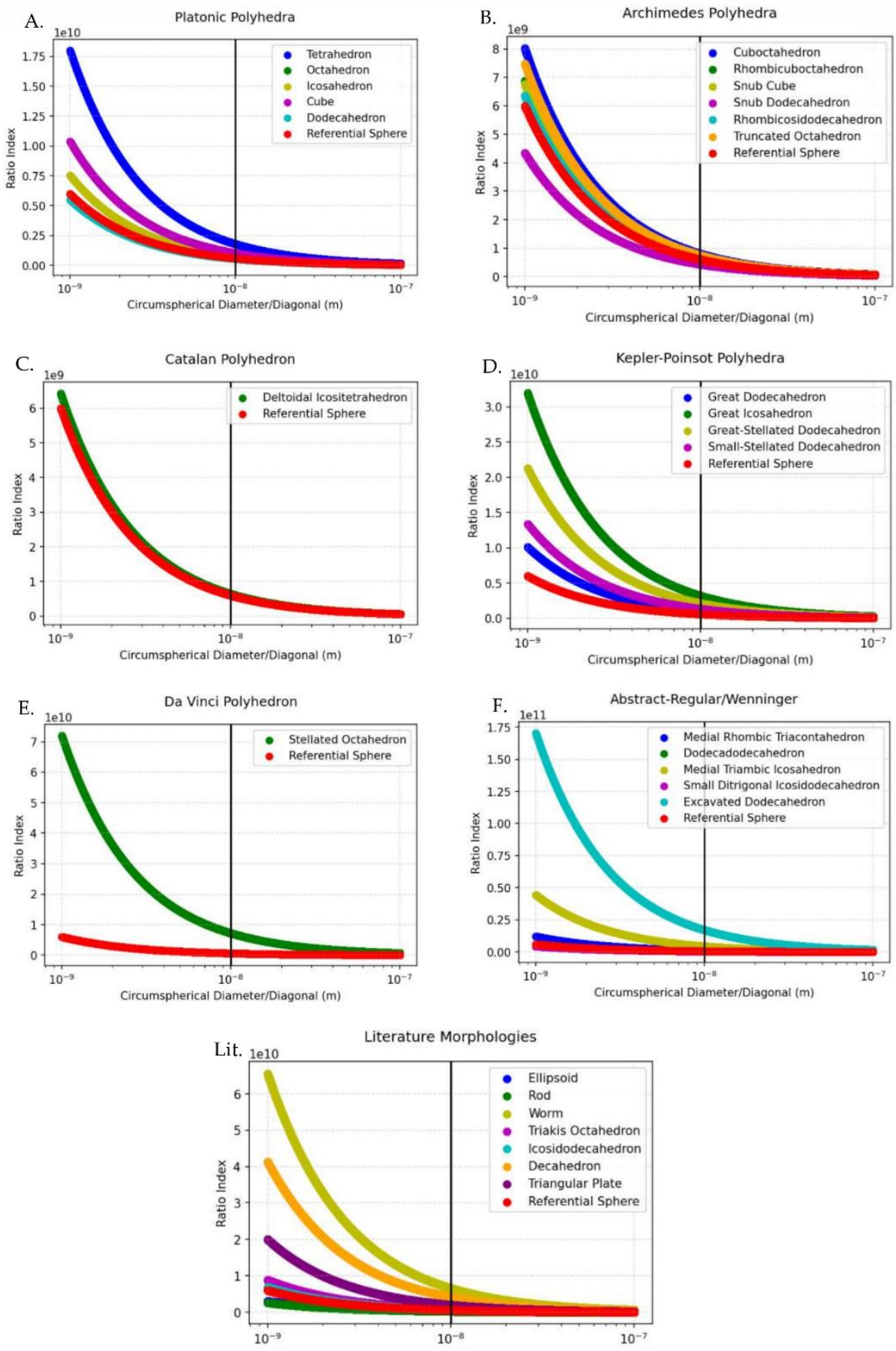

Figure 3. Six-panel scatter plot of $\mathrm{SA}: \mathrm{V}$, measured in $\mathrm{m}^{-1}$, as a function of morphology across the nanoregime for the A. Platonic, B. Archimedes, C. Catalan, D. Kepler-Poinsot, E. Da Vinci, F. Wenninger, and Lit. Literature solids/polyhedra. *Note the multiplicative scaling factor is given at the top of the SA:V axis. 
Table 5. Unpaired two-sample $t$ tests performed on five groups and globally of the investigated polyhedra with a sample size at least 5: A. Platonic, B. Archimedes/Catalan, C. Kepler-Poinsot, Da Vinci, D. Wenninger polyhedra, Lit. Literature Solids, and Global: Global t-test. Critical SA:V denotes the threshold of statistical significance with respect to $\mathrm{SA}: \mathrm{V}$ (based on t-test statistic equation) solely as a rationale for choosing superior shapes within a group $\left({ }^{*}=\mathrm{p}<.05\right)$.

\begin{tabular}{|c|c|c|}
\hline Parameters (A.) & \multirow{2}{*}{\multicolumn{2}{|c|}{ Shapes Sphere (ref.) }} \\
\hline Mean & 1.04 & \\
\hline Variance & 0.22. & 0 \\
\hline Observations & 5 & 5 \\
\hline Hypothesized Mean Difference & $\mathrm{c}$ & \\
\hline df & 4 & \\
\hline $\mathrm{t}$ Stat & 2.06 & \\
\hline $\mathrm{P}(\mathrm{T}>=\mathrm{t})$ one-tail & 0.05 & \\
\hline tCritical one-tail & 2.13 & \\
\hline Critical SA:V & 1.05 & \\
\hline Parameters (B.) & \multicolumn{2}{|c|}{ Shapes Sphere (ref.) } \\
\hline Mean & $0.6 \mathrm{C}$ & 0.6 \\
\hline Variance & 0.01 & 0 \\
\hline Observations & ? & \\
\hline Hypothesized Mean Difference & c & \\
\hline df & te & \\
\hline tStat & 1.4 & \\
\hline $\mathrm{P}(\mathrm{T}>\mathrm{t})$ one-tail & 0.11 & \\
\hline$t$ Critical one-tail & 1.9 & \\
\hline Critical SA:V & 0.65 & \\
\hline
\end{tabular}

\begin{tabular}{|c|c|c|}
\hline Parameters (C.) & \multicolumn{2}{|c|}{ Shapes Sphere (ref.) } \\
\hline Mean & 3.04 & 0.6 \\
\hline Variance & 5.98 & \\
\hline Observations & 5 & 5 \\
\hline Hypothesized Mean Difference & 0 & \\
\hline df & 4 & \\
\hline $\mathrm{t}$ Stat & 2.23 & \\
\hline $\mathrm{P}(\mathrm{T}>=\mathrm{t})$ one-tail & 0.04 & \\
\hline$t$ Critical one-tail & 2.13 & \\
\hline Critical SA:V & 2.93 & \\
\hline Parameters (D.) & \multicolumn{2}{|c|}{ Shapes Sphere (ref.) } \\
\hline Mean & 4.7 & 0.6 \\
\hline Variance & 50.02 & 0 \\
\hline Observations & 5 & 5 \\
\hline Hypothesized Mean Difference & 0 & \\
\hline df & 4 & \\
\hline tStat & 1.3 & \\
\hline$P(T>=t)$ one-tail & 0.13 & \\
\hline t Critical one-tail & 2.13 & \\
\hline Critical SA:V & 7.36 & \\
\hline
\end{tabular}

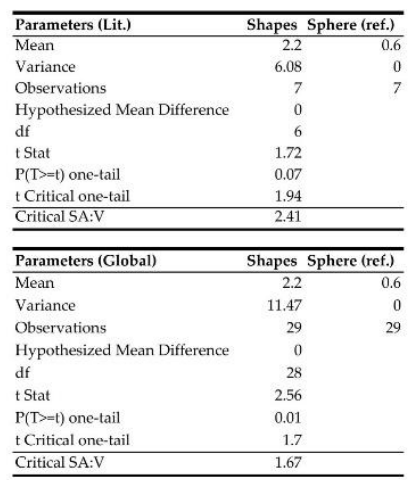

\section{Conclusions}

Only 9 out of 29 investigated shapes were significant in regard to the global t-test and only 9 out of 22 (different array) were seen as significant within the groups. The convex group exhibits a lack of effect in the global t-test only showcasing significance amongst its low numbers while the global $\mathrm{t}$-test indicates more significance in the concave group, showing how lower variance in potentially high significance in the groups can mar determination of shapes best for experimentation. Many were either on the cusp of significance or not at all, showing that some may offer little rationale behind their choice while others would show little to no efficacy at all based on the SA:V parameter alone. Multiple other parameters may make a shape attractive for investigation as does any highly controlled object of experimentation. The shapes that possess this significance, however, warrant investigation first in basic research for inorganic nanoparticles employing the best or most available methods for creating complex structures or molds from multiple processing methods. More complex and possibly efficacious morphologies may require more specialized nanofabrication. Obviously, some may not be feasible to develop, but they could offer, in addition to the others, a possible novel open inquiry of investigation as well.

The state-of-the-art in nanotechnology is ever evolving and refining. This paper showcases one of those examples in regard to the morphological tuning of inorganic nanoparticles as a promising aspect of optimization in nanomedicine and nanotechnology itself. A refinement that acknowledges long-discovered aspects of advanced geometry, mathematical derivation/proofing, and computer programming further proves the uncanny ability for this field to be amenable and tenable to being interdisciplinary, which continues to serve its constant need for updating, well.

Supplementary Materials: The following are available online at $w w w . m d p i . c o m / x x x / s 1$

Author Contributions: Conceptualization, W.A.W., and D.J.D; methodology, W.A.W.; software, W.A.W.; validation, W.A.W.; formal analysis, W.A.W. A,D., P.R. ; investigation, W.A.W.; resources, W.A.W.; data curation, W.A.W.; writing - original draft preparation, W.A.W.; writing - review and editing, D.J.D. and S.S. M; visualization, W.A.W.; supervision, S.S.M.; project administration, S.S.M.; funding acquisition, S.S.M. All authors have read and agreed to the published version of the manuscript.

Funding: This research was supported by the Graduate Program Research Support to students, Taneja College of Pharmacy, University of South Florida. SSM is a Veteran Affairs Research Career Scientist (IK6BX003778) and his research is supported by a VA Merit Review Program (BX003685).

Acknowledgments: W.A.W. thanks Ashley Denslow and Peter Radulovic for their assistance with Python syntax and to Glärbo, from the Mathematics section of the website: Stack Exchange, who helped with the general SA and V derivations for certain unique polyhedra. 
Conflicts of Interest: The authors declare no conflict of interest.

\section{References}

[1] Mohapatra, S. S., Frisina, R. D., Mohapatra, S., Sneed, K. B., Markoutsa, E., Wang, T., Dutta, R., Damnjanovic, R., Phan, M. H., Denmark, D. J., Biswal, M. R., McGill, A. R., Green, R., Howell, M., Ghosh, P., Gonzalez, A., Ahmed, N. T., Borresen, B., Farmer, M., . . Martin, D. K. (2020). Advances in Translational Nanotechnology: Challenges and Opportunities. Applied Sciences, 10(14), 4881. https://doi.org/10.3390/app10144881

[2] Shin, M. D., Shukla, S., Chung, Y. H., Beiss, V., Chan, S. K., Ortega-Rivera, O. A., Wirth, D. M., Chen, A., Sack, M., Pokorski, J. K., \& Steinmetz, N. F. (2020). COVID-19 vaccine development and a potential nanomaterial path forward. Nature Nanotechnology, 15(8), 646-655. https://doi.org/10.1038/s41565-020-0737-y

[3] Denmark, D. J., Bustos-Perez, X., Swain, A., Phan, M. H., Mohapatra, S., \& Mohapatra, S. S. (2019). Readiness of Magnetic Nanobiosensors for Point-of-Care Commercialization. Journal of Electronic Materials, 48(8), 47494761. https://doi.org/10.1007/s11664-019-07275-7

[4] Giner-Casares, J. J., Henriksen-Lacey, M., Coronado-Puchau, M., \& Liz-Marzán, L. M. (2016). Inorganic nanoparticles for biomedicine: where materials scientists meet medical research. Materials Today, 19(1), 19-28. https://doi.org/10.1016/j.mattod.2015.07.004

[5] Denmark, D. J., Hyde, R. H., Gladney, C., Phan, M. H., Bisht, K. S., Srikanth, H., Mukherjee, P., \& Witanachchi, S. (2017). Photopolymerization-based synthesis of iron oxide nanoparticle embedded PNIPAM nanogels for biomedical applications. Drug Delivery, 24(1), 1317-1324. https://doi.org/10.1080/10717544.2017.1373164

[6] Denmark, D. J., Bradley, J., Mukherjee, D., Alonso, J., Shakespeare, S., Bernal, N., Phan, M. H., Srikanth, H., Witanachchi, S., \& Mukherjee, P. (2016). Remote triggering of thermoresponsive PNIPAM by iron oxide nanoparticles. RSC Advances, 6(7), 5641-5652. https://doi.org/10.1039/c5ra21617f

[7] Khodashenas, B., \& Ghorbani, H. R. (2019). Synthesis of silver nanoparticles with different shapes. Arabian Journal of Chemistry, 12(8), 1823-1838. https://doi.org/10.1016/j.arabjc.2014.12.014

[8] Navya, P. N., \& Daima, H. K. (2016). Rational engineering of physicochemical properties of nanomaterials for biomedical applications with nanotoxicological perspectives. Nano Convergence, 3(1). https://doi.org/10.1186/s40580-016-0064-z

[9] Nemati, Z., Alonso, J., Martinez, L. M., Khurshid, H., Garaio, E., Garcia, J. A., Phan, M. H., \& Srikanth, H. (2016). Enhanced Magnetic Hyperthermia in Iron Oxide Nano-Octopods: Size and Anisotropy Effects. The Journal of Physical Chemistry C, 120(15), 8370-8379. https://doi.org/10.1021/acs.jpcc.6b01426

[10] Wu, Z., Yang, S., \& Wu, W. (2016). Shape control of inorganic nanoparticles from solution. Nanoscale, 8(3), 1237-1259. https://doi.org/10.1039/c5nr07681a

[11] Thambi, V., Kar, A., Ghosh, P., Paital, D., Gautam, A. R. S., \& Khatua, S. (2019). Synthesis of Complex Nanoparticle Geometries via pH-Controlled Overgrowth of Gold Nanorods. ACS Omega, 4(9), $13733-13739$. https://doi.org/10.1021/acsomega.9b01119

[12] Lee, Y. H., Lay, C. L., Shi, W., Lee, H. K., Yang, Y., Li, S., \& Ling, X. Y. (2018). Creating two self-assembly microenvironments to achieve supercrystals with dual structures using polyhedral nanoparticles. Nature Communications, 9(1). https://doi.org/10.1038/s41467-018-05102-x

[13] Shen, J., Shafiq, M., Ma, M., \& Chen, H. (2020). Synthesis and Surface Engineering of Inorganic Nanomaterials Based on Microfluidic Technology. Nanomaterials, 10(6), 1177. https://doi.org/10.3390/nano10061177

[14] Eguchi, M., Mitsui, D., Wu, H. L., Sato, R., \& Teranishi, T. (2012). Simple Reductant Concentration-Dependent Shape Control of Polyhedral Gold Nanoparticles and Their Plasmonic Properties. Langmuir, 28(24), 9021-9026. https://doi.org/10.1021/la3002114

[15] Polavarapu, L., Mourdikoudis, S., Pastoriza-Santos, I., \& Pérez-Juste, J. (2015). Nanocrystal engineering of noble 
metals and metal chalcogenides: controlling the morphology, composition and crystallinity. CrystEngComm, 17(20), 3727-3762. https://doi.org/10.1039/c5ce00112a

[16] Biswas, A., Bayer, I. S., Biris, A. S., Wang, T., Dervishi, E., \& Faupel, F. (2012). Advances in top-down and bottom-up surface nanofabrication: Techniques, applications \& future prospects. Advances in Colloid and Interface Science, 170(1-2), 2-27. https://doi.org/10.1016/j.cis.2011.11.001

[17] Ben-Akiva, E., Meyer, R. A., Yu, H., Smith, J. T., Pardoll, D. M., \& Green, J. J. (2020). Biomimetic anisotropic polymeric nanoparticles coated with red blood cell membranes for enhanced circulation and toxin removal. Science Advances, 6(16), eaay9035. https://doi.org/10.1126/sciadv.aay9035

[18] Andre Conjusteau, A. L. (2015). Biocompatible Gold Nanorod Conjugates for Preclinical Biomedical Research. Journal of Nanomedicine \& Nanotechnology, 06(02). https://doi.org/10.4172/2157-7439.1000274

[19] Cancino-Bernardi, J., Marangoni, V., Besson, J., Cancino, M., Natali, M., \& Zucolotto, V. (2018). Gold-based nanospheres and nanorods particles used as theranostic agents: An in vitro and in vivo toxicology studies. Chemosphere, 213, 41-52. https://doi.org/10.1016/j.chemosphere.2018.09.012

[20] Ridolfo, R., Tavakoli, S., Junnuthula, V., Williams, D. S., Urtti, A., \& van Hest, J. C. M. (2020). Exploring the Impact of Morphology on the Properties of Biodegradable Nanoparticles and Their Diffusion in Complex Biological Medium. Biomacromolecules, 22(1), 126-133. https://doi.org/10.1021/acs.biomac.0c00726

[21] Yan, L., Mu, J., Ma, P., Li, Q., Yin, P., Liu, X., Cai, Y., Yu, H., Liu, J., Wang, G., \& Liu, A. (2021). Gold nanoplates with superb photothermal efficiency and peroxidase-like activity for rapid and synergistic antibacterial therapy. Chemical Communications, 57(9), 1133-1136. https://doi.org/10.1039/d0cc06925f

[22] Zhou, S., Zhao, M., Yang, T. H., \& Xia, Y. (2019). Decahedral nanocrystals of noble metals: Synthesis, characterization, and applications. Materials Today, 22, 108-131. https://doi.org/10.1016/j.mattod.2018.04.003

[23] Chien, Y. H., Huang, C. C., Wang, S. W., \& Yeh, C. S. (2011). Synthesis of nanoparticles: sunlight formation of gold nanodecahedra for ultra-sensitive lead-ion detection. Green Chemistry, 13(5), 1162. https://doi.org/10.1039/c0gc00915f

[24] Anselmo, A. C., \& Mitragotri, S. (2019). Nanoparticles in the clinic: An update. Bioengineering \& Translational Medicine, 4(3). https://doi.org/10.1002/btm2.10143

[25] NBTXR3 Development. (n.d.). Nanobiotix.Retrieved August 21, 2021, from https://www.nanobiotix.com/nbtxr3-development/

[26] Bonvalot, S., le Pechoux, C., de Baere, T., Kantor, G., Buy, X., Stoeckle, E., Terrier, P., Sargos, P., Coindre, J. M., Lassau, N., Ait Sarkouh, R., Dimitriu, M., Borghi, E., Levy, L., Deutsch, E., \& Soria, J. C. (2016). First-in-Human Study Testing a New Radioenhancer Using Nanoparticles (NBTXR3) Activated by Radiation Therapy in Patients with Locally Advanced Soft Tissue Sarcomas. Clinical Cancer Research, 23(4), 908-917. https://doi.org/10.1158/1078-0432.ccr-16-1297

[27] Kummer, J. K. (n.d.). Geometric Shapes and Solids - Geometry Calculator. Recheronline. Retrieved August 21, 2021, from https://rechneronline.de/pi/geometry.php

[28] Webb, Robert "Stella: Polyhedron Navigator," Symmetry: Culture and Science, Vol. 11, Nos. 1-4, p231268, 2000 (available online at http://www.software3d.com/PolyNav/PolyNavigator.php)

[29] Cauchy, A. L. "Recherches sur les polyèdres." J. de l'École Polytechnique 9, 68-86, 1813.

[30] Wenninger, M. J. (1971). Polyhedron Models. Cambridge University Press.

[31] S. Torquato and Y. Jiao, "Dense packings of the Platonic and Archimedean solids," Nature, vol. 460, no. 7257, pp. 876-879, Aug. 2009, doi: 10.1038/nature08239.

[32] Pacioli, L., \&amp; Marinoni, A. (1982). De divina proportione. Silvano.

[33] Catalan, E. (1865). Mémoire sur la théorie des polyèdres (Vol. 24). Gauthier-Villars (Journal: l'École Polytechnique). 
[34] Deng, H., Dutta, P., \& Liu, J. (2019). Entry modes of ellipsoidal nanoparticles on a membrane during clathrinmediated endocytosis. Soft Matter, 15(25), 5128-5137. https://doi.org/10.1039/c9sm00751b

[35] Moreau, L. M., Jones, M. R., Roth, E. W., Wu, J., Kewalramani, S., O’Brien, M. N., Chen, B. R., Mirkin, C. A., \& Bedzyk, M. J. (2019). The role of trace Ag in the synthesis of Au nanorods. Nanoscale, 11(24), 11744-11754. https://doi.org/10.1039/c9nr03246k

[36] Truong, N. P., Quinn, J. F., Whittaker, M. R., \& Davis, T. P. (2016). Polymeric filomicelles and nanoworms: two decades of synthesis and application. Polymer Chemistry, 7(26), 4295-4312. https://doi.org/10.1039/c6py00639f

[37] Ma, Y., Kuang, Q., Jiang, Z., Xie, Z., Huang, R., \& Zheng, L. (2008). Synthesis of Trisoctahedral Gold Nanocrystals with Exposed High-Index Facets by a Facile Chemical Method. Angewandte Chemie, 120(46), 9033-9036. https://doi.org/10.1002/ange.200802750

[38] Fujita, D., Ueda, Y., Sato, S., Yokoyama, H., Mizuno, N., Kumasaka, T., \& Fujita, M. (2016). Self-Assembly of M 30 L 60 Icosidodecahedron. Chem, 1(1), 91-101. https://doi.org/10.1016/j.chempr.2016.06.007

[39] Bharti, S., Mukherji, S., \& Mukherji, S. (2021). Enhanced antibacterial activity of decahedral silver nanoparticles. Journal of Nanoparticle Research, 23(2). https://doi.org/10.1007/s11051-020-05106-z

[40] Wijaya, Y. N., Kim, J., Choi, W. M., Park, S. H., \& Kim, M. H. (2017). A systematic study of triangular silver nanoplates: one-pot green synthesis, chemical stability, and sensing application. Nanoscale, 9(32), 11705-11712. https://doi.org/10.1039/c7nr03077k 